\title{
DNA methylation as a sensitive biomarker of environmental abiotic factor exposure
}

\author{
Minasbekyan L.A. ${ }^{1 *}$, Aidarkhanova G.S. ${ }^{2}$, Avagyan I.A. ${ }^{3}$ \\ ${ }^{1}$ Yerevan State University, Research Institute of Biology, Yerevan, Armenia \\ ${ }^{2}$ S. Seifullin Kazakh AgroTechnical University, Nur-Sultan, Kazakhstan \\ ${ }^{3}$ SRC of V\&TC at AM of RA, Darakert, Armenia \\ *e-mail:minlia@ysu.am
}

The great number of biotic and abiotic factors affect plant under natural growing conditions growth. DNA sequence does not carry the complete information necessary to determine the phenotype of the organism. DNA methylation controls genomic integration, regulates genome expression and cell differentiation, as well as plant response to biotic and abiotic stresses. Epigenetic regulation involves various reversible chemical modifications occurring on both the DNA itself and the proteins interacting with it, which as a result affects the chromatin structure and function, without, however, altering the sequence of nucleic residues in the DNA.

We have previously shown on wheat seedlings Tr. aestivum (v. Nairi) the importance of the role of DNA methylation in the formation of a response to abiotic stress, as well as the transformation of these changes into the next generation. The results of our research have proved once again that DNA methylation is a sensitive biomarker for the environmental impact of environmental factors. However, in order to understand the role of epigenetic changes in the adaptation and evolution of plants, require further studies of DNA methylation on the model-based Tr. aestivum, A. thaliana, O. sativa, Z. mays, and on non-model plants.

Now special importance is attached to the study of the population of pasture plants, since environmental pollution through animal food can be transmitted to human. Due to these circumstances, it is imperative to regularly monitor pasture plants, both for relocating animals to more environmentally friendly meadows, and environmental protection measures aimed at improving damaged pastures. Among pasture plants Bromus inermis, Medicago sativa, Onobrychis arenaria, Agropyron pectinoforme, etc. are widely distributed. Currently we have studied the DNA methylation of esparcet - Onobrichis arenaria and Agropyron pectinoforme. The obtained data on epigenetic changes on the studied plants will improve the methods of pasture monitoring, taking into account the ecological, climatic conditions of the regions and the agricultural sector of the countries' economies. 